\title{
Interplay of STDP and Dendritic Plasticity in a Hippocampal CA1 Pyramidal Neuron Model
}

\author{
Ausra Saudargiene ${ }^{1,2}$, Rokas Jackevicius ${ }^{2}$, and Bruce P. Graham ${ }^{3}$ \\ ${ }^{1}$ Neuroscience Institute, Lithuanian University of Health Sciences, Kaunas, Lithuania \\ ${ }^{2}$ Department of Informatics, Vytautas Magnus University, Kaunas, Lithuania \\ ${ }^{3}$ Division of Computing Science \& Mathematics, \\ School of Natural Sciences, University of Stirling, Stirling, United Kingdom \\ ausra.saudargiene@lsmuni.lt
}

\begin{abstract}
Synaptic plasticity in hippocampal CA1 pyramidal neurons is accompanied and shaped by dendritic plasticity, the long-lasting and region-specific alteration of the biophysical properties of voltage-gated dendritic ion channels. Down-regulation of A-type potassium current $I_{\mathrm{A}}$, observed after long-term potentiation induction, boosts the amplitude of somatic back-propagating action potentials and the associated calcium concentration in dendritic spines, increases the amplitude of the excitatory postsynaptic potentials and promotes synaptic integration. Using a detailed computational model of a CA1 pyramidal cell and a spiketiming-dependent synaptic plasticity (STDP) protocol we found that supression of A-type potassium current $I_{\mathrm{A}}$ leads to the increased dendritic excitability converting long-term depression to long-term potentiation in proximal synapses and supporting fast Hebbian plasticity in distal synapses on a hippocampal CA1 pyramidal neuron.
\end{abstract}

Keywords: Spike-timing-dependent Synaptic Plasticity, Dendritic Plasticity, A-type Potassium Current $I_{\mathrm{A}}$, CA1 Pyramidal Neuron

\section{Introduction}

Biological neurons store memories not only in their synapses, but also in the biophysical properties of dendritic ion channels. Long-term spike-timing-dependent synaptic plasticity (STDP) refers to the synaptic ability to modify its strenght depending on the temporal order of presynaptic and postsynaptic activity: if the presynaptic spike precedes the postsynaptic spike within a short time window, the synapse undergoes long-term potentiation (LTP), and it exhibits long-term depression (LTD) if the temporal order is reversed [2]. Physiological experiments show that induction of long-term synaptic plasticity is followed by a local increase in dendritic excitability in CA1 pyramidal neurons [4], [8], [9], [11]. Specifically, LTP is accompanied by a region-specific and long-lasting down-regulation of Atype potassium current $I_{\mathrm{A}}$ that leads to the boosted amplitude of the somatic back-propagating action potentials (bAPs), elevated calcium levels in dendrites 
and dendritic spines, increased amplitude of the excitatory postsynaptic potentials and enchanced synaptic integration [4]. Decreased $I_{\mathrm{A}}$ allows effective backpropagation of somatic action potentials into the distal dendrites and promotes LTP induction in distal synapses [3].

In this study we analyzed the influence of dendritic plasticity on the properties of synaptic plasticity in a hippocampal CA1 pyramidal neuron using a computational modeling approach. We assumed that LTP was induced at synapses on proximal apical dendrites of a CA1 pyramidal neuron and supressed A-type potassium current $I_{\mathrm{A}}$ in proximal dendritic region. We modeled synaptic weight changes in proximal stratum radiatum (SR) and distal lacunosum-moleculare (SLM) spines of a CA1 pyramidal neuron, applying a classical STDP stimulation protocol. The results show that $I_{\mathrm{A}}$ - mediated dendritic plasticity enchances learning in the CA1 pyramidal neuron.

\section{Methods}

\subsection{Model of CA1 Pyramidal Neuron}

We use a detailed compartmental model of CA1 pyramidal neuron [7] with the spines on the proximal SR dendrite and distal SLM dendrite $98 \mu \mathrm{m}$ and $324 \mu \mathrm{m}$ from the soma, respectively (Fig. 1). The model includes a leak current, somatic/axonic and dendritic Hodgkin-Huxley-type sodium and potassium currents, proximal and distal A-type potassium currents, m-type potassium current, a mixed conductance hyper polarization-activated h-current, low-voltageactivated T-type calcium current, somatic and dendritic high-voltage-activated R-type currents, somatic and dendritic high-voltage-activated L-type currents, two types of $\mathrm{Ca}^{2+}$-dependent potassium currents (a slow afterhyperpolarization current and a medium afterhyperpolarization current), and a persistent sodium current. Full details of the model are given in [7]. Spines contain the same ion channels as their parent dendrites. Spine head diameter and length equal $0.5 \mu \mathrm{m}$, spine neck diameter is $0.2 \mu \mathrm{m}$ and length is $1 \mu \mathrm{m}$. Dendritic spines express AMPA and NMDA receptor-gated synaptic channels modeled via a conventional double exponential function, including $M g^{2+}$ block for NMDA channels. Peak AMPA synaptic conductance is $1.5 \mu S$, and peak NMDA synaptic conductance is $0.003 \mu S$.

The stimulation protocol consists of the presynaptic action potential at the SR spine or SLM spine paired with a burst-like doublet of somatic action potentials [10], schematically shown in Fig. 1. Postsynaptic action potentials in doublets are spaced $10 \mathrm{~ms}$ apart. $T$ denotes a temporal difference between the presynaptic and a second postsynaptic action potential: if the presynaptic action potential precedes a second postsynaptic potential, $T$ is positive; if the temporal order is reversed, $T$ is negative. $T$ values are set to $-10 \mathrm{~ms}$ and $10 \mathrm{~ms}$. Stimulation consists of 100 pairings at $5 \mathrm{~Hz}$ and lasts $20 \mathrm{~s}$ as in experimental study[10]. 

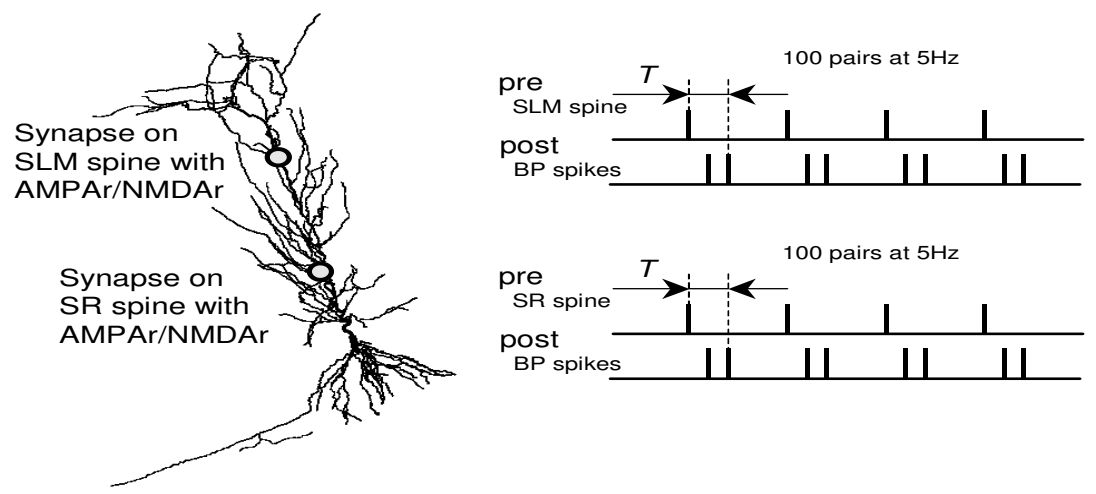

Fig. 1. Compartmental model of a CA1 pyramidal neuron [7] with the added AMPA/NMDA receptor-gated channels on the proximal stratum radiatum (SR) and distal lacunosum-moleculare (SLM) spines, and schematic illustration of the stimulation protocols. Presynaptic action potential at the SR spine and SLM spine is paired with a burst-like doublet of somatic action potentials spaced $10 \mathrm{~ms}$ apart. $T$ denotes a temporal difference between the presynaptic and a second postsynaptic action potential and is set to $-10 \mathrm{~ms}$ and $10 \mathrm{~ms}$.

\subsection{Synaptic and Dendritic Plasticity in a CA1 Pyramidal Neuron Model}

Synaptic modifications are driven by the intracellular calcium concentration in the SR and SLM spines. Calcium concentration in spines is modelled as [1]:

$$
\frac{\mathrm{d}\left[\mathrm{Ca}^{2+}\right]}{\mathrm{d} t}=-\frac{I_{C a}}{2 F d 18}+\frac{\left[\mathrm{Ca}^{2+}\right]_{0}-\left[\mathrm{Ca}^{2+}\right]}{\tau_{C a}},
$$

where $\left[\mathrm{Ca}^{2+}\right]$ is the calcium concentration in a spine, $I_{C a}$ is the calcium current into the spine, $\left[\mathrm{Ca}^{2+}\right]_{0}=0.1 \mu \mathrm{M}$ is the resting calcium concentration in a spine, factor 18 reflects the influence of the endogenous buffers [1], $\tau_{C a}=15 \mathrm{~ms}$ is the time constant of calcium concentration decay [1], $F$ is the Faraday constant, $d=0.1 \mu m$ is the depth of dendritic shell.

Synaptic efficacy variable $\rho$ is described by a differential equation [5]:

$\tau \frac{\mathrm{d} \rho}{\mathrm{d} t}=-\rho(1-\rho)\left(\rho_{0}-\rho\right)+\gamma_{\mathrm{p}}(1-\rho) \Theta\left(\left[C a^{2+}\right]-\theta_{\mathrm{p}}\right)-\gamma_{\mathrm{d}} \rho \Theta\left(\left[C a^{2+}\right]-\theta_{\mathrm{d}}\right)+N$ oise $(t)$,

where $\left[\mathrm{Ca}^{2+}\right]$ is the instantaneous calcium concentration in a spine, $\rho_{0}=0.5$ is unstable $\rho$ state, $\gamma_{\mathrm{p}}=400$ is the rate of increase in $\rho, \gamma_{\mathrm{d}}=100$ is the rate of decrease in $\rho, \theta_{\mathrm{p}}=0.8$ is the LTP threshold, $\theta_{\mathrm{d}}=0.24$ is the LTD threshold, $\Theta$ is the the Heaviside function, Noise $(t)$ is an activity-dependent noise term (not implemented), $\tau=100 \mathrm{~s}$ is the time constant of $\rho$ changes.

In SR and SLM spines $\rho$ is set to the DOWN and to the UP states $\rho_{D O W N}$ and $\rho_{U P}$ to represent either an initially unpotentiated or a potentiated synapse. 
Transitions from the DOWN to the UP or from the UP to the DOWN states trigger positive or negative synaptic weight change [5]:

$$
\Delta \omega=-1+\frac{((1-U) \beta+D(1-\beta))+b(U \beta+(1-D)(1-\beta))}{\beta+(1-\beta) b},
$$

where $U$ is the probability of $\rho_{D O W N}$ transition from the DOWN state to the UP state, $D$ is the probability of $\rho_{U P}$ transition from the UP state to the DOWN state, $\beta=0.7$ is the fraction of synapses in the DOWN state, $b=5$ is the synaptic strength of the UP/DOWN state. Initially, $U=0$ and $D=0$; during learning $U$ = becomes 1 when $\rho_{D O W N}$ increases and crosses the 0.5 threshold; $D=1$ when $\rho_{U P}$ decreases and crosses the 0.5 threshold.

The numerical simulations of the compartmental model of a CA1 pyramidal neuron were performed in NEURON [6].

\section{Results}

Presynaptic action potential at a SR spine, paired with a doublet of postsynaptic action potentials at $T=-10 \mathrm{~ms}$ and $T=10 \mathrm{~ms}$, leads to LTD and LTP as observed in physiological experiments [10] (Fig. 2). If the presynaptic action potential follows the second postsynaptic spike by $T=-10 \mathrm{~ms}$ (Fig. 2a), calcium concentration in a SR spine reaches $1.8 \mu M$ (Fig. 2b) and leads to synaptic efficacy variable $\rho$ transition from the UP state to the DOWN state (Fig. 2c, black line) and negative synaptic weight change, i.e. LTD induction (Fig. 2d). If the presynaptic action potential precedes the second postsynaptic spike by $T=10 \mathrm{~ms}$ (Fig. 2e), calcium concentration in a SR spine increases up to $2.2 \mu \mathrm{M}$ (Fig. 2f) and forces synaptic efficacy variable $\rho$ to transit from the DOWN state to the UP state (Fig. 2g, gray line) leading to the positive weight change, i.e. LTP induction (Fig. 2h).

The experiments were repeated under the influence of the enchanced local excitability of the proximal apical dendrites of a CA1 pyramidal neuron (Fig. $3 \mathrm{~g}$ ). The voltage of half-maximal inactivation for A-type potassium current was hyperpolarised from $-63 m V$ to $-69 m V$, and maximal conductance of A-type potassium channel was down-scaled by a factor of 0.2 in the SR apical dendrites up to $121 \mu \mathrm{M}$ from the soma to account for the experimentally observed increase in the amplitude of bAPs and dendritic calcium concentration. For $T=-10 \mathrm{~ms}$, calcium levels in a SR spine are elevated up to $2.2 \mu M$ (Fig. 3b), synaptic efficacy variable $\rho$ performs transition from the DOWN state to the UP state (Fig. 3c, gray line) and leads to the positive synaptic weight change and LTP induction (Fig. 3d). For $T=10 \mathrm{~ms}$, calcium in a SR spine reaches higher values of $3.1 \mu \mathrm{M}$ (Fig. 3f) and induces LTP (Fig. 3g, h).

Similar stimulation protocol was applied for the distal SLM spine: presynaptic action potential at a SLM spine was paired with a doublet of postsynaptic somatic action potentials at $T=-10 \mathrm{~ms}$ and $T=10 \mathrm{~ms}$. In control conditions, synaptic modifications are not induced for $T=-10 \mathrm{~ms}$ (not shown); a SLM synapse is depressed for $T=10 \mathrm{~ms}$ (Fig. 4 , a-d) as the bAPs are too weak to 
invade distal dendritic regions and effectively open NMDA receptor-gated channels that mediate strong calcium current, necessary for LTP. If A-type potassium current is down-regulated, bAPs, paired with the SLM synaptic input, induce dendritic spikes for $T=-10 \mathrm{~ms}$ (not shown) and for $T=10 \mathrm{~ms}$ (Fig. 4e, black line). Dendritic spikes open NMDA receptor-gated channels and contribute to the elevation of intracellular calcium concentration in a SLM spine via voltagegated calcium channels. Calcium concentration reaches $37 \mu M$ and causes fast LTP (Fig. 4g, black line; h)

(a) $V_{m}$ in SR spine and soma

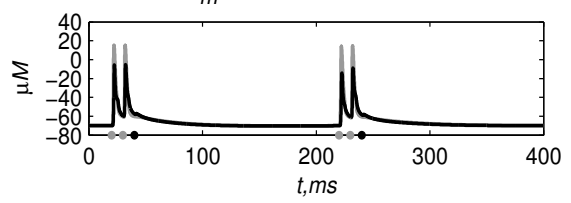

(b) $\left[\mathrm{Ca}^{2+}\right]$ in SR spine

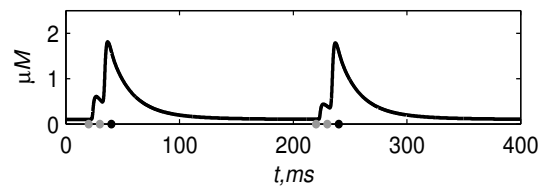

(c) Synaptic efficacy $\rho$ in SR spine

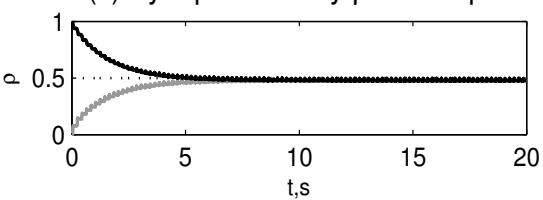

(d) Synaptic weight change in SR spine

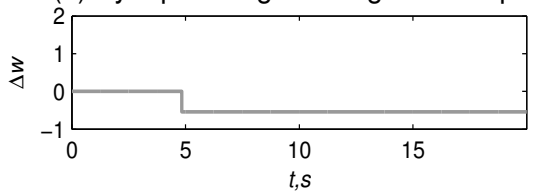

(e) $V_{m}$ in SR spine and soma

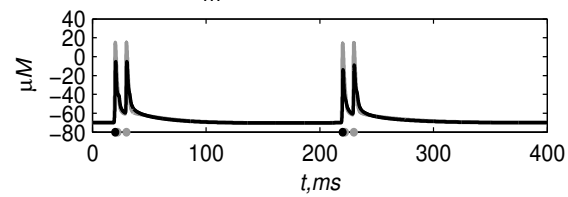

(f) $\left[\mathrm{Ca}^{2+}\right]$ in SR spine

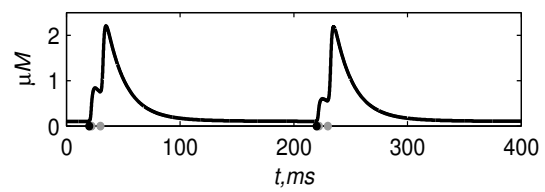

(g) Synaptic efficacy $\rho$ in SR spine

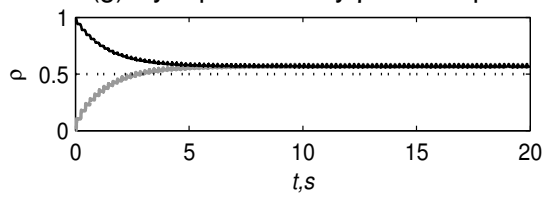

(h) Synaptic weight change in SR spine

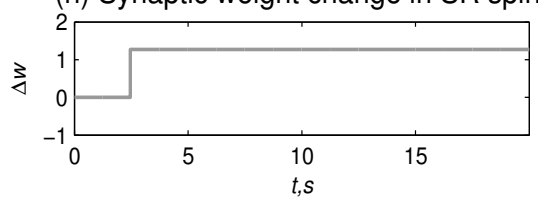

Fig. 2. Membrane potential, calcium concentration, synaptic efficacy and synaptic weight change in a proximal SR spine for $T=-10 \mathrm{~ms}$ (a)-(d) and $T=10 \mathrm{~ms}$ (e)(h) in control conditions. (a,e) Membrane potential in a proximal SR spine (black line) and soma (gray line) during two pre-post pairings. Postsynaptic action potentials are generated at $20 \mathrm{~ms}$ and $30 \mathrm{~ms} ; 220 \mathrm{~ms}$ and $230 \mathrm{~ms}$ (gray dots). Every second postsynaptic action potential in a burst is used as a reference point for $T$ definition. Presynaptic action potential is induced at $40 \mathrm{~ms}$ and at $240 \mathrm{~ms}$ for $T=-10 \mathrm{~ms}$; at $20 \mathrm{~ms}$ and at $220 \mathrm{~ms}$ for $T=10 \mathrm{~ms}$ (black dots). (b,f) Calcium concentration in a proximal SR spine during two pre-post pairings. $(\mathrm{c}, \mathrm{g})$ Synaptic efficacy $\rho$ : initially in DOWN state - gray line; initially in UP state - black line. $(\mathrm{d}, \mathrm{h})$ Synaptic weight change: $(\mathrm{d})$ for $T=-10 \mathrm{~ms}$ LTD is induced; (h) for $T=10 \mathrm{~ms}$ LTP is induced. 
(a) $V_{m}$ in SR spine and soma

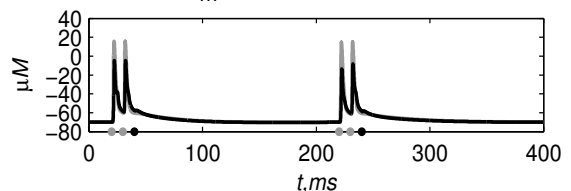

(b) $\left[\mathrm{Ca}^{2+}\right]$ in SR spine

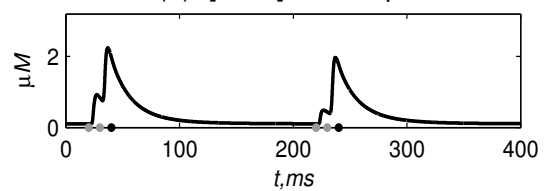

(c) Synaptic efficacy $\rho$ in SR spine

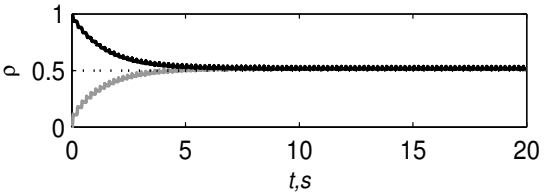

(d) Synaptic weight change in SR spine

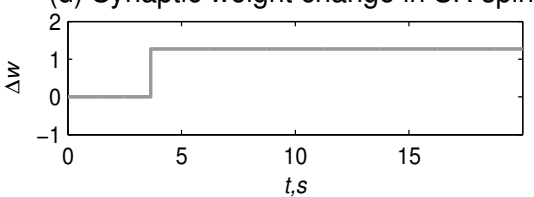

(e) $V_{m}$ in SR spine and soma

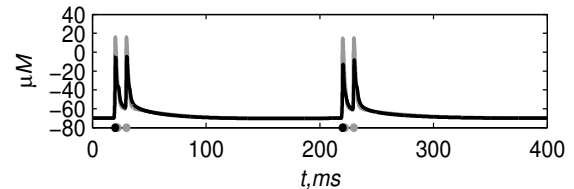

(f) $\left[\mathrm{Ca}^{2+}\right]$ in SR spine

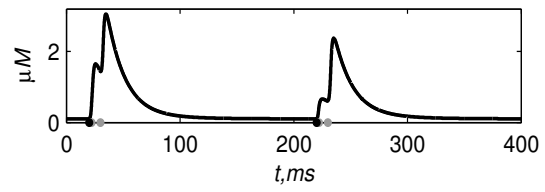

(g) Synaptic efficacy $\rho$ in SR spine

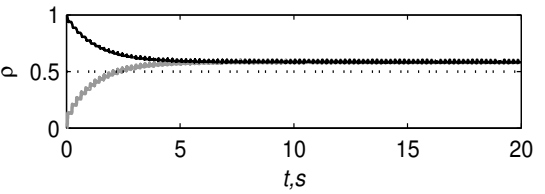

(h) Synaptic weight change in SR spine

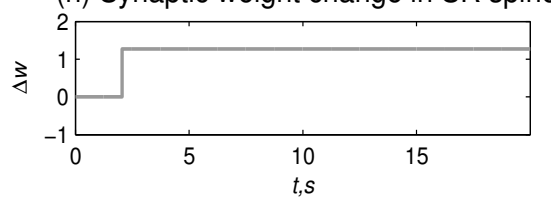

Fig. 3. Membrane potential, calcium concentration, synaptic efficacy and synaptic weight change in a proximal SR spine for $T=-10 \mathrm{~ms}$ (a)-(d) and $T=10 \mathrm{~ms}$ (e)-(h) in conditions of enhanced local excitability of proximal dendritic region. (a,e) Membrane potential in a proximal SR spine (black line) and soma (gray line) during two pre-post pairings. Stimulation protocol as in Fig. 2. (b,f) Calcium concentration in a proximal SR spine during two pre-post pairings. $(c, g)$ Synaptic efficacy $\rho$ initially in DOWN state - gray line; initially in UP state - black line. $(\mathrm{d}, \mathrm{h})$ Synaptic weight change: $(\mathrm{d})$ for $T=-10 \mathrm{~ms}$ LTP is induced; (h) for $T=10 \mathrm{~ms}$ LTP is induced. 
(a) $V_{m}$ in SLM spine and soma

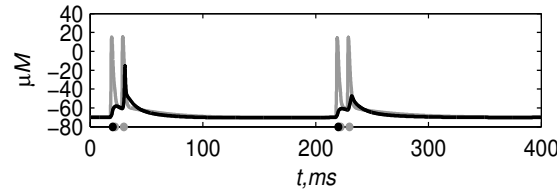

(b) $\left[\mathrm{Ca}^{2+}\right]$ in SLM spine

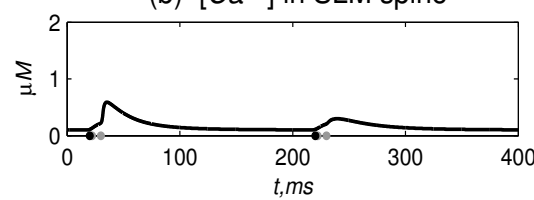

(c) Synaptic efficacy $\rho$ in SLM spine

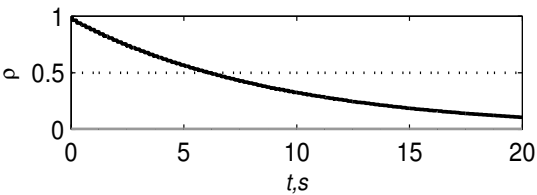

(d) Synaptic weight change in SLM spine

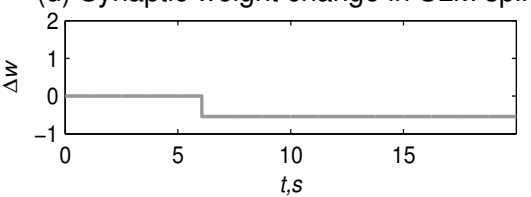

(e) $V_{m}$ in SLM spine and soma

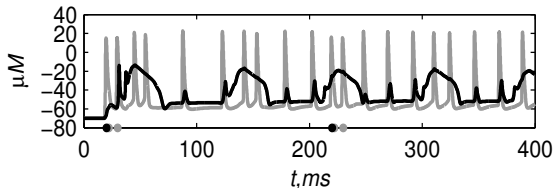

(f) $\left[\mathrm{Ca}^{2+}\right]$ in SLM spine

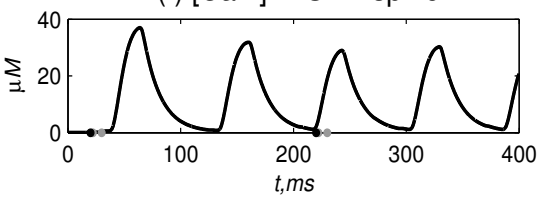

(g) Synaptic efficacy $\rho$ in SLM spine

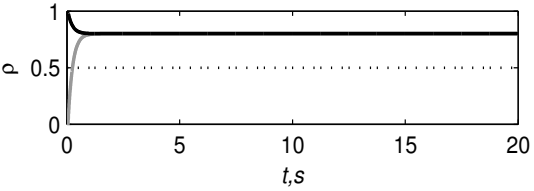

(h) Synaptic weight change in SLM spine

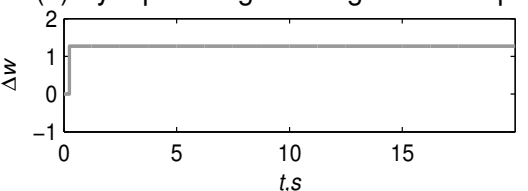

Fig. 4. Membrane potential, calcium concentration, synaptic efficacy and synaptic weight change in a distal SLM spine for $T=10 \mathrm{~ms}$ in control conditions (a)-(d) and in conditions of enhanced local excitability of proximal dendritic region (e)-(h). (a,e) Membrane potential in a distal SLM spine (black line) and soma (gray line) during two pre-post pairings. Presynaptic action potential is induced at $20 \mathrm{~ms}$ and at $220 \mathrm{~ms}$ (black dots). In control conditions (a), the postsynaptic action potentials are generated at $20 \mathrm{~ms}$ and $30 \mathrm{~ms} ; 220 \mathrm{~ms}$ and $230 \mathrm{~ms}$ (gray dots), every second postsynaptic action potential is used as a reference point for $T$ definition. In conditions of enhanced dendritic excitability (e), back-progating action potentials paired with the distal synaptic input induce dendritic spikes that propagate to the soma and cause somatic action potentials. $(\mathrm{b}, \mathrm{f})$ Calcium concentration in a distal SLM spine during two pre-post pairings. $(c, g)$ Synaptic efficacy $\rho$ initially in DOWN state - gray line; initially in UP state - black line. (d,h) Synaptic weight change: (d) LTD is induced; (h) fast LTP is induced. 


\section{Conclusions}

Long-lasting region-specific down-regulation of A-type potassium current $I_{\mathrm{A}}$ is an important factor of synaptic plasticity in hippocampal CA1 pyramidal neuron as it boosts the amplitude of the somatic back-propagating action potentials and the associated calcium concentration in SR spines, and creates favorable conditions for dendritic spike generation in distal SLM dendritic regions. Simulation results show that supression of $I_{\mathrm{A}}$ acts as a switch from LTD to LTP in proximal SR synapses (Fig. 2,d and Fig. 3,d) and supports fast Hebbian plasticity in distal SLM spines due to dendritic spike initiation (Fig. 4,e,h). Dendritic plasticity, following long-term potentiation induction, serves as a positive feedback for synaptic plasticity and promotes reliable and fast learning. Biological learning rules might serve as an inspiration for more efficient learning algorithms in spiking neural networks, neuromorphic computing and other brain-inspired artificial neural systems.

\section{References}

1. Badoual, M., Zou, Q., Davison, A.P., Rudolph, M., Bal, T., Fregnac, Y., Destexhe, A.: Biophysical and phenomenological models of multiple spike interactions in spike-timing dependent plasticity. Int J Neural Syst 16(2), 79-97 (Apr 2006)

2. Bi, G., Poo, M.: Synaptic modification by correlated activity: Hebb's postulate revisited. Annu. Rev. Neurosci. 24, 139-166 (2001)

3. Chen, X., Yuan, L., Zhao, C., Birnbaum, S., Frick, A., Jung, W., Schwarz, T., Sweatt, J., Johnston, D.: Deletion of Kv4.2 gene eliminates dendritic A-type K+ current and enhances induction of long-term potentiation in hippocampal CA1 pyramidal neurons. J.Neurosci. 26(47), 12143-12151 (Nov 2006)

4. Frick, A., Magee, J., Johnston, D.: LTP is accompanied by an enhanced local excitability of pyramidal neuron dendrites. Nat. Neurosci. 7(2), 126-135 (Feb 2004)

5. Graupner, M., Brunel, N.: Calcium-based plasticity model explains sensitivity of synaptic changes to spike pattern, rate, and dendritic location. Proc. Natl. Acad. Sci. U.S.A. 109(10), 3991-3996 (Mar 2012)

6. Hines, M., Carnevale, N.: The NEURON simulation environment. Neural Comput. 9(6), 1179-1209 (1997)

7. Poirazi, P., Brannon, T., Mel, B.: Arithmetic of subthreshold synaptic summation in a model CA1 pyramidal cell. Neuron 37, 977-987 (Aug 2003)

8. Sjostrom, P.J., Hausser, M.: A cooperative switch determines the sign of synaptic plasticity in distal dendrites of neocortical pyramidal neurons. Neuron 51(2), 227 238 (Jul 2006)

9. Wang, Z., Xu, N.L., Wu, C.P., Duan, S., Poo, M.M.: Bidirectional changes in spatial dendritic integration accompanying long-term synaptic modifications. Neuron 37(3), 463-472 (Feb 2003)

10. Wittenberg, G.M., Wang, S.S.: Malleability of spike-timing-dependent plasticity at the CA3-CA1 synapse. J. Neurosci. 26(24), 6610-6617 (Jun 2006)

11. Xu, J., Kang, N., Jiang, L., Nedergaard, M., Kang, J.: Activity-dependent longterm potentiation of intrinsic excitability in hippocampal CA1 pyramidal neurons. J. Neurosci. 25(7), 1750-1760 (Feb 2005) 